PROCEEDINGS OF THE

AMERICAN MATHEMATICAL SOCIETY

Volume 131, Number 6, Pages 1789-1791

S 0002-9939(02)06759-X

Article electronically published on October 1, 2002

\title{
SIMILARITY TO AN ISOMETRY OF A COMPOSITION OPERATOR
}

\author{
FRÉDÉRIC BAYART
}

(Communicated by N. Tomczak-Jaegermann)

\begin{abstract}
We study the composition operators which are similar to an isometry on the classical Hardy space $H^{2}(\mathbb{D})$.
\end{abstract}

\section{INTRODUCTION}

Throughout this paper, we denote by $\mathbb{D}$ the open unit disc in the complex plane, by $\mathbb{T}$ the circle, and by $m$ the Haar measure on $\mathbb{T}$. We also denote by $H(\mathbb{D})$ the space of holomorphic functions on $\mathbb{D}$, and by $H(\mathbb{D}, \mathbb{D})$ the subset of $H(\mathbb{D})$ consisting of all self-maps of $\mathbb{D}$.

We recall that the Hardy Space $H^{2}(\mathbb{D})$ is the subspace of $H(\mathbb{D})$ consisting of all functions $f$ satisfying

$$
\|f\|_{2}:=\left(\sup _{0 \leq r<1} \int_{0}^{2 \pi} \frac{1}{2 \pi}\left|f\left(r e^{i \theta}\right)\right|^{2} d \theta\right)^{1 / 2}<+\infty .
$$

Every function $f \in H^{2}(\mathbb{D})$ has a radial limit, i.e.,

$$
\lim _{r \rightarrow 1^{-}} f\left(r e^{i \theta}\right)=f^{*}\left(e^{i \theta}\right)
$$

exists for almost every $e^{i \theta} \in \mathbb{T}$. Furthermore, we have $f^{*} \in L^{2}$, and $\|f\|_{H^{2}(\mathbb{D})}=$ $\left\|f^{*}\right\|_{L^{2}}$. In the sequel, we will write $f$ instead of $f^{*}$. A function $\phi \in H(\mathbb{D}, \mathbb{D})$ is said to be inner if $\left|\phi\left(e^{i t}\right)\right|=1$ almost everywhere.

Let $\phi \in H(\mathbb{D}, \mathbb{D})$. We define on $H^{2}(\mathbb{D})$ the composition operator induced by $\phi$ by $C_{\phi}(f)=f \circ \phi$. It is well-known that $C_{\phi}(f) \in H^{2}(\mathbb{D})$, and that $C_{\phi}$ is continuous on $H^{2}(\mathbb{D})$. More precisely, the following inequalities hold (see [2], p. 123):

$$
\left(\frac{1}{1-|\phi(0)|^{2}}\right)^{1 / 2} \leq\left\|C_{\phi}\right\| \leq\left(\frac{1+|\phi(0)|}{1-|\phi(0)|}\right)^{1 / 2}
$$

In particular, $C_{\phi}$ is a contraction if and only if $\phi(0)=0$. Moreover, in Nordgren's classical paper [6], it is shown that if $\phi(0)=0$ and if $\phi$ is inner, then $C_{\phi}$ is an isometry. Our aim in this paper is to prove the following theorem:

Theorem 1. Let $\phi \in H(\mathbb{D}, \mathbb{D})$. The following are equivalent:

i) $\phi$ is inner, and has a fixed point in $\mathbb{D}$.

ii) $C_{\phi}$ is similar to an isometry.

Received by the editors October 9, 2001 and, in revised form, January 16, 2002.

2000 Mathematics Subject Classification. Primary 47B38, 46B22.

Key words and phrases. Similarity to an isometry, composition operator, Hardy space. 
This result was first shown by N.Jaoua ([4]) under some additional assumptions on the symbol ( $\phi$ is analytic in a neighborhood of the closed disk). He uses a result of Kamowitz on spectra of composition operators, and a weak form of the DenjoyWolff theorem. It turns out that, by a recent result of J.Shapiro ([7]), no extra assumption on $\phi$ is needed. Indeed, Shapiro proved: Suppose that $\phi$ is an analytic self map of the disk fixing the origin. If $\phi$ is not inner, then the restriction of $C_{\phi}$ to the subspace of functions in $H^{2}$ which are 0 at 0 has norm strictly less than 1 . Therefore, it is sufficient to prove that $\phi$ has a fixed point as soon as $C_{\phi}$ is similar to an isometry. We will give a proof of this fact later.

The aim of this paper is to give a simple and self-contained proof of Theorem 1 which can be extended to other situations, such as composition operators on Hardy spaces of Dirichlet series. With minor modifications, the same arguments apply, for example, in Theorem 3.5 of [1].

\section{Proof of the MAin theorem}

We recall Nagy's criterion ( $[5]$ ) of similarity to an isometry. We will just use the easier implication.

Theorem 2 (Nagy). $S$ is similar to an isometry if and only if

$$
\exists k>0, \forall x \in X, \forall n \in \mathbb{N}, \frac{1}{k}\|x\| \leq\left\|S^{n} x\right\| \leq k\|x\| .
$$

In other words, $S$ is power-bounded, and the ranges of $S^{n}$ are uniformly closed.

Cima, Thomson and Wogen proved in $\left[3\right.$ that the composition operator $C_{\phi}$ on $H^{2}(\mathbb{D})$ has closed range if and only if

$$
\exists c>0, \forall B \subset \mathbb{T}, m\left(\phi^{-1}(B) \cap \mathbb{T}\right) \geq c m(B),
$$

where $B$ is a Borel set of $\mathbb{T}$ and $\phi^{-1}(B)=\{z \in \overline{\mathbb{D}}, \phi(z) \in B\}$. On the other hand, a composition operator is injective, so it has closed range if and only if there exists $k>0$ such that, for all $f \in H^{2}(\mathbb{D})$, we have $k\|f\| \leq\left\|C_{\phi}(f)\right\|$. In the next lemma, we use arguments similar to those in [3] to give a quantitative relation between $c$ and $k$.

Lemma 1. Let $C_{\phi}$ be a composition operator on $H^{2}(\mathbb{D})$, and $k>0$ be a positive constant. We suppose that, for all $f \in H^{2}(\mathbb{D})$, we have $k\|f\| \leq\left\|C_{\phi}(f)\right\|$. Then, for every Borel set $B \subset \mathbb{T}$, one has

$$
m\left(\phi^{-1}(B) \cap \mathbb{T}\right) \geq k^{2} m(B) .
$$

Proof. Let $B$ be such a set, and $A=\phi^{-1}(B) \cap \mathbb{T}$. We take a function $f \in H^{\infty}(\mathbb{D})$ such that

$$
|f|= \begin{cases}1 & \text { on } B \\ 1 / 2 & \text { on } \mathbb{T}-B .\end{cases}
$$

The existence of such a function follows from Beurling's theory of outer functions. It is clear that $\left\|f^{n}\right\|_{H^{2}}^{2} \rightarrow m(B)$. On the other hand, we have

$$
\left\|C_{\phi}\left(f^{n}\right)\right\|_{H^{2}}^{2}=\int_{A}\left|f^{n} \circ \phi\right|^{2} d m+\int_{\mathbb{T}-A}\left|f^{n} \circ \phi\right|^{2} d m .
$$

But, $\int_{A}\left|f^{n} \circ \phi\right|^{2} d m=m(A)$, whereas $|f \circ \phi(z)|<1$ for $z \in \mathbb{T}-A$. Lebesgue's theorem then implies that

$$
\left\|C_{\phi}\left(f^{n}\right)\right\|^{2} \rightarrow m(A)
$$


Passing to the limit in the inequality $k^{2}\left\|f^{n}\right\|^{2} \leq\left\|C_{\phi}\left(f^{n}\right)\right\|^{2}$, we get that $m(A) \geq$ $k^{2} m(B)$.

Our principal result follows from this lemma:

Proof of Theorem 1. We already know that if $\phi$ is inner and $\phi(0)=0$, then $C_{\phi}$ is an isometry. Now, if $\phi(a)=a$, let $\psi$ be an automorphism of $\mathbb{D}$ which maps $a$ to 0 . Let $\varphi=\psi^{-1} \circ \phi \circ \psi$. Then $C_{\varphi}$ is an isometry. $C_{\phi}$, which is similar to $C_{\varphi}$, is in particular similar to an isometry.

Conversely, suppose that $C_{\phi}$ is similar to an isometry. Let $\phi_{j}$ be the function $\phi \circ \cdots \circ \phi$ ( $j$ times). By the assumption, there exist $K, k>0$ such that

$$
\forall j \in \mathbb{N}, \forall f \in H^{2}(\mathbb{D}), K\|f\| \geq\left\|C_{\phi}^{j}(f)\right\|=\left\|C_{\phi_{j}}(f)\right\| \geq k\|f\| .
$$

Now, suppose that $C_{\phi}$ has no fixed point in $\mathbb{D}$. By a weak form of the Denjoy-Wolff theorem, we have $\left|\phi_{n}(0)\right| \rightarrow 1$. Therefore

$$
\left\|C_{\phi}^{n}\right\|=\left\|C_{\phi_{n}}\right\| \geq\left(1-\left|\phi_{n}(0)\right|\right)^{-1 / 2} .
$$

This inequality contradicts (2). We now prove that $\phi$ is inner. Using the lemma, for all Borel sets $B$ of $\mathbb{T}$, and all $j \in \mathbb{N}$, we have $m\left(\phi_{j}^{-1}(B) \cap \mathbb{T}\right) \geq k^{2} m(B)$. If $\phi$ were not inner, there would exist $B \subset \mathbb{T}$ such that $m(B)>0$, and $|\phi(z)|<1$ if $z \in B$. Let us define $B_{0}=B$, and $B_{j}=\phi_{j}^{-1}(B) \cap \mathbb{T}$. We have by Lemma 1 ;

$$
m\left(B_{j}\right) \geq k^{2} m(B)
$$

We now observe that the $B_{j}$ 's are disjoint:

$$
\text { If } i \neq j, B_{i} \cap B_{j}=\varnothing \text {. }
$$

Indeed, if for example $i<j$ and $z \in B_{i}, \phi_{i}(z) \in B$, so $\left|\phi_{j}(z)\right|=\left|\phi_{j-i}\left(\phi_{i}(z)\right)\right|<1$. As $B \subset \mathbb{T}, \phi_{j}(z) \notin B$, and this implies $z \notin B_{j}$. But it follows from (44) that $\sum m\left(B_{j}\right) \leq m(\mathbb{T}) \leq 1$, and this fact is in contradiction with (3). This ends the proof of Theorem 1 .

\section{REFERENCES}

[1] F. Bayart, De nouveaux espaces de séries de Dirichlet et leurs opérateurs de composition, CRAS 333 (2001), 207-212 MR 2002f:47051

[2] C.C Cowen and B.D. MacCluer, Composition Operators on Spaces of Analytic Functions, CRC Press, Boca Raton (1995) MR 97i:47056

[3] J.Cima, J.Thomson, W. Wogen, On some properties of Composition Operators, Ind. Math. Journal 24 (1974), 215-221 MR 50:2979

[4] N.Jaoua, Similarity to a contraction and hypercontractivity of composition operators, Proceedings of the American Math. Society 129 (2001), 2085-2092 MR 2002d:47038

[5] B.Sz. Nagy, On uniformly bounded linear transformations in Hilbert space. Acta Univ. Szeged (1947)

[6] E.Nordgren, Composition operators, Can. J. Math. 20 (1968), 442-449 MR 36:6961

[7] J.H. Shapiro, What do composition operators know about inner functions?, Monat Math 130 (2000), 57-70 MR 2001a:47029

UStl, Laboratoire AGAT, U.F.R. de Mathématiques, 59665 Villeneuve d’AscQ Cedex, FRANCE

E-mail address: bayart@agat.univ-lille1.fr 